\title{
DIGITgame: Gamification as Amazing way to learn STEM Concepts Developing Sustainable cities Idea in the Citizen of the Future
}

\author{
Laura Bonora \\ National Research Council, Institute of Biometeorology \\ Francesca Martelli \\ National Research Council, Institute of Biometeorology \\ Valentina Marchi \\ National Research Council, Institute of Biometeorology
}

\begin{abstract}
DIGITgame, intends to improve scientific skills and competences through an innovative teaching methods. It is based on Smart City projecting and realized by the development of a mini-game with challenges based on the scientific concepts. DIGITgame proposes a new approach to teach traditional topics of environmental sciences using the thematic of Smart Cities and taking advantage introducing the videogame solution. It will permit to reinforce the achievement of advanced and high quality competences, both in scientific topics and digital knowledge. Match between traditional teaching approach, gamification challenge and informatics is the keystone to turn students in involved and aware citizens.
\end{abstract}

Keywords: STEM, Gamification, Smart City

\section{INTRODUCTION}

DIGITgame is a project founded by the E.U. in the framework of Erasmus+ program, axis KA2 'Cooperation for Innovation and the Exchange of Good Practices' and objective 'KA201 Strategic Partnerships for school education'.

This generation is immersed in interactive, immediate and multimodal communication mediated through technology. As a result, many educational researchers have asserted that digital natives have different ways of expressing themselves and different learning preferences because of their immersion with ICT. (Tapscott, 1998; Prensky, 2010).

Despite our increasing technological dependence, the decline in high quality scientific vocations is inexorable. The EC studied this trend and raised concerns about it. Kier (Kier, et al., 2014) stated that negative attitudes and lack of interest about Science, Engineering and Mathematic skills are two of the main problems to be addressed facing the loss interest of youth in increasing and consolidating their school knowledge. Many high school students see school science as uninteresting, unimportant and irrelevant to their lives, as a matter of rote learning fact (Kirsti, 2007). 
Regarding science education, students often fail simple questions because they hold misconceptions or use and interlink concepts incorrectly and this hinders their domain related reasoning. Research findings suggest that traditional instructional approaches like textbook based and hands-on practice often do not succeed in deep conceptual understanding.

Digital natives students have different learning styles and preferences (Sue, 2008) characterized by a multitasking approach, oriented to visual media; they prefer to work on activities rather than reading texts or following instruction, and are less motivated in the environments that lack technology. Because of this, it is crucial that schools and educators respond to this students' experiences in ways that are significant for education; teachers can leverage against technologies allowing students to meet demands of a future workforce in a global information society.

The DIGITgame project intends to attract young people to science studies by supplying an alternative way to use and apply basic and advanced scientific concepts in a technological tool very familiar and appealing as a videogame. The positive competition between the different "designer groups" in different school and countries will increase the use of acquired concepts to develop alternative ideas and solutions to better face the game contest.

\section{METHOD: INITIAL SITUATION DEFINITION AND DIDACTIC STRATEGY DESIGNING}

In this section, we describe how the DIGITgame didactic approach, in facing STEM underachievement and lack of interest impact, has been documented using a pre-and post testing strategy and how the cross-subject compared analysis between IT and TK science issues has been carried out as strategic teaching platform for the successive project actions.

These first results represent a mix of quantitative monitoring (questionnaires) and qualitative case study methodology.

\section{Initial Situation Definition}

The first steps required to determine how the general problem of the teaching STEM with innovative didactic strategies is to find a way to translate into a number the initial hypotheses concerning the student consciousness in technology for learning.

A formal standardized questionnaire is a survey instrument useful to collect data from individuals about themselves, their habits and behaviour. Based on that, the impact of DIGITgame didactic approach will be documented using a pre-and post testing strategy; method popular (even if a bit controversial in literature), easy to apply and characterize by a not time-consuming application for students and teachers (low dropout rate). The method, based on targeted questionnaires, permits to check student's and teacher's changes particularly in attitudes and behaviour toward science specific subject and related learning/teaching strategies. With the pre/post-testing investigation, students and teachers are asked to share the knowledge or attitude they had toward particular aspects in approaching teaching/learning science before DIGITgame experience and after. In general, when participants are asked to respond to a question about how much they are conscious about a particular behaviour, they are unconsciously more able to accurately reflect, during and after specific and aimed actions (DIGITgame activities), on the degree of change in knowledge or attitude.

Furthermore, respondents oftentimes are not aware on behaviours or mindset in specific daily and usual contexts. With the retrospective questionnaires respondents are given an opportunity to fell and to test how much they face considered issues by a passive attitude.

With these aims DIGITgame foresee three types of questionnaire;

- an initial set of questions to investigate the approach and behaviour, both of students and teachers, in considering and using devices (Smartphone, PC, tablet) and Internet support in investigating science subjects,

- a set of questions, shared at the end of the project operative activities, to understand the changes in devices and Internet utility awareness, 
- a final questionnaire to test the effective level of appreciation and fun experienced by the project actors during the activities and in general in the project participation.

The main aim of "questionnaires actions" in DIGITgame project is to understand the impact of the activities and resources and efforts exploited. Concerning the reference methodology we decide to apply the approach proposed by Kier et al. (Kier, et al., 2014)) in a simplified version fitted to the project activities and deadlines.

In the project the "questionnaire activity" represented a small survey aimed to give indications related to attitudinal, habits and preferences, it isn't a systematic data collection concerning student's information across all of the STEM subject areas. This kind of survey contributes to develop measurements and investigations toward STEM context and high school students' interest in STEM.

The questionnaires were administrated to 113 students and 23 teachers.

A questionnaire template has been developed based on the adaptation of the 'Research and Science Education" questionnaire.

It is realized to achieve the following results:

- Identify the knowledge on STEM

- Identify the use of smartphone to study

- Identify the level of knowledge of science topics that will be increased during the project.

The same questionnaires, with some changes, will be administrated at the end of the pilot activity to understand and compare the level of knowledge before and at the project end. The template of the questionnaire has been planned in different sections to detect different aspects. The survey results were displayed in simple descriptive statistical tables and graphs. (See chapter First results)

\section{Didactic Strategy Designing}

The first efforts to define the project didactic strategy have been driven by the little consciousness about the differences in school curricula between EU national institutions.

Many descriptions are available regarding the purposes of a comparative education and didactic structure analysis such as understanding our own and other countries' educational systems; improving, developing, and reforming educational systems, policy, and practice; predicting the success and consequences of educational change; and developing tools to aid the construction of theoretical frameworks (Songül, 2009).

Few studies of teaching strategy have examined the differences in teacher effectiveness are related to differences in subject matter covered, and there is still a tendency to discuss issues of teaching and learning in general terms separated from the content that has been taught.

In this framework, project Consortium carried out the TK and IT school curriculum collection and comparison in order to have the necessary indications on common subjects and issues. In collaboration with the partner schools, the age's groups and the specific strands and subject - characterizing the focus of the two STEM National School curricula - the curricula has been identified in detail and then summarized in main skill for an easier reading and comparison.

School curricula in science can be approached and analyzed in different ways; including the broad areas of knowledge (concepts) to be covered; evaluating the specific activities to be carried out; focusing learning outcomes to be achieved (the skills that have to be acquired).

The DIGITgame team strategy to meet the specific project objective has been to consider the national range of science learning and issues to merge finally in a selection of subject that will contribute to more than one learning outcome.

After this comparison activity, the team identified a core set of issues derived by the previous matching on common STEM subjects in IT and TK national school curricula. These issues have been classified in two main groups: Variables and Assets (See Table 1). This general grouping is functional to the successive didactic work and organization. Variables constitute the game software structure that will be developed by the virtual world software Company; it is the general framework where students will develop the Assets. In fact, by the selection of climate/industrialization/green and transport levels, the 
game identifies the final objective: increase or maintain the Smart level of the city. It is possible by an intelligent use of the Assets in the game.

Concerning the assets these have been focused as the main STEM common elements that will be the subject of a double deepening.

1) traditional lessons: on each Asset, a specific traditional lesson has been defined and structured, by an operative worksheet; in this way it will be possible to replicate lessons in different classes. The traditional lesson is tuned to supply students detailed information concerning the Science, Technology, Engineering and Mathematic elements that we want to reinforce in their scholastic schooling.

2) videogame creation: this second didactic step will engage students in practical application related to the traditional lessons learned in the previous phase of the project. They have to build the game logic by the inclusion of Assets (recycled station, tree, green transport etc.) in the game developments.

For each asset score, costs, pro and cons have to be defined with the aims to build our City (defined by the Variable selection) as Smart as possible.

The innovative didactic idea is to involve students in build and tuning the videogame logic by making use of the scientific knowledge acquired in the point 1.

TABLE 1

VARIABLES AND ASSETS

\begin{tabular}{|l|l|}
\hline VARIABLES & $\begin{array}{l}\text { ASSETS (Realized by pilot classrooms in Italy } \\
\text { and Turkey) }\end{array}$ \\
\hline $\begin{array}{l}\text { Climate - Three Types: Dry and windy / Hot and } \\
\text { humid / Humid and cold (no seasons - yearly } \\
\text { average) }\end{array}$ & $\begin{array}{l}\text { Recycle Stations (they will buy and place them } \\
\text { once the answer some questions) - related to } \\
\text { Smart City }\end{array}$ \\
\hline $\begin{array}{l}\text { Industrialization - Three Types: Low / Medium / } \\
\text { High }\end{array}$ & $\begin{array}{l}\text { Trees (10 from Italy - 10 from Turkey) (some } \\
\text { features of assets will be set as unchangeable) - } \\
\text { related to VOC }\end{array}$ \\
\hline Level of Green: Low / Medium / High & $\begin{array}{l}\text { Weather Stations: One basic - One more advance } \\
\text { (students will answer questions to modify their } \\
\text { station from basic to advance) }\end{array}$ \\
\hline Green Transport: Yes or Not & $\begin{array}{l}\text { Questions about green transport and cars to } \\
\text { increase money or score }\end{array}$ \\
\hline & $\begin{array}{l}\text { Solar Panels (the energy that is produced increases } \\
\text { the score. The solar panels have to be bought) }\end{array}$ \\
\hline & $\begin{array}{l}\text { Smart Building (elements - for a fee - to build the } \\
\text { house to increase the score and happiness) }\end{array}$ \\
\hline
\end{tabular}

After the identification of assets and variables the software company involved in the project identified the platform that support the mini-game and developed the variables. They have set three kind of places: small village, medium town and large city. 
FIGURE 1

\section{DIGITGAME PLACES RENDERING}

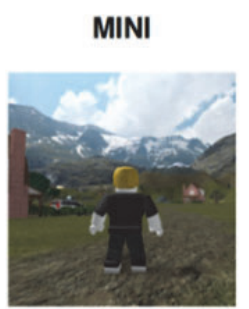

SMALL VILLAGE

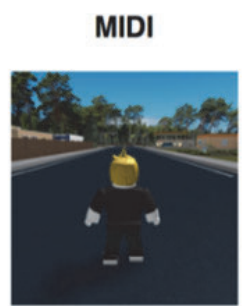

MEDIUM TOWN
MAXI

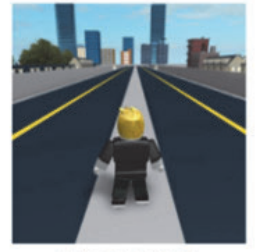

LARGE CITY

The user manual on the platform, realized by the company, is the main tool used by the first group of students to create assets and design their Smart Cities (See Fig. 1.). Students with the support of teachers have defined the elements to insert in the city and the score to be given to each created asset (See Fig. 2).

FIGURE 2

DIGITGAME ASSETS EXAMPLE

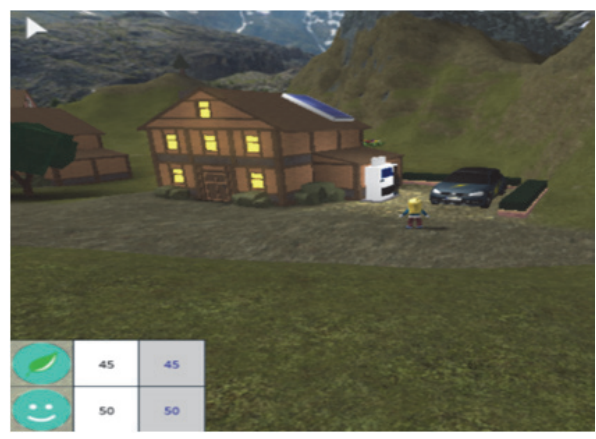

\section{FIRST RESULTS}

The Initial situation definition survey results are here displayed in simple descriptive statistical tables and graphs. In the first part of the questionnaire, same general information about young students will be collected, such as age, gender, school. In Figure 3 the results about gender from Italian and Turkish schools. We immediately see a balanced percentage between males $(58 \%)$ and females (42\%) who completed the questionnaire. 


\section{FIGURE 3}

\section{GENDER DISTRIBUTION}

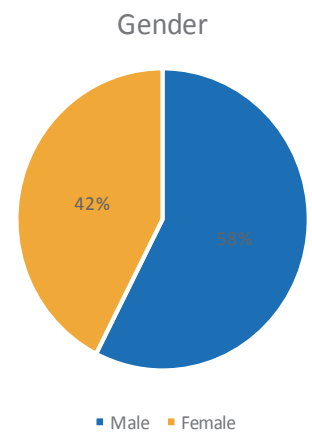

The second section of the questionnaire detects the knowledge on skills in relation to Science, Technology, Engineering and Mathematics (15 questions). Data were collected through a Likert-type test scheme (Likert, 1932) based on a five-value scale: $1=$ strongly disagree; $2=$ disagree; $3=$ neither agree nor disagree; 4=agree; $5=$ strongly agree.

The following figure (See Fig. 4) shows the results related to the level of knowledge and interaction with the STEM subjects. Students were involved to answer questions about some aspects on STEM with refers on the subject class, homework, future career and the importance of new technology to improve and deepen topics treated at school. The aim of this section is to detect the different vision on STEM skills and use of smartphone/ICT from students. The results underline that males and females have given answers fairly balanced for all items. The main item with a marked different score from male and female is related to the question 'I use the smartphone/pc to increase topics treated at school'. The highest score was given by males. 
FIGURE 4

STEM SKILLS
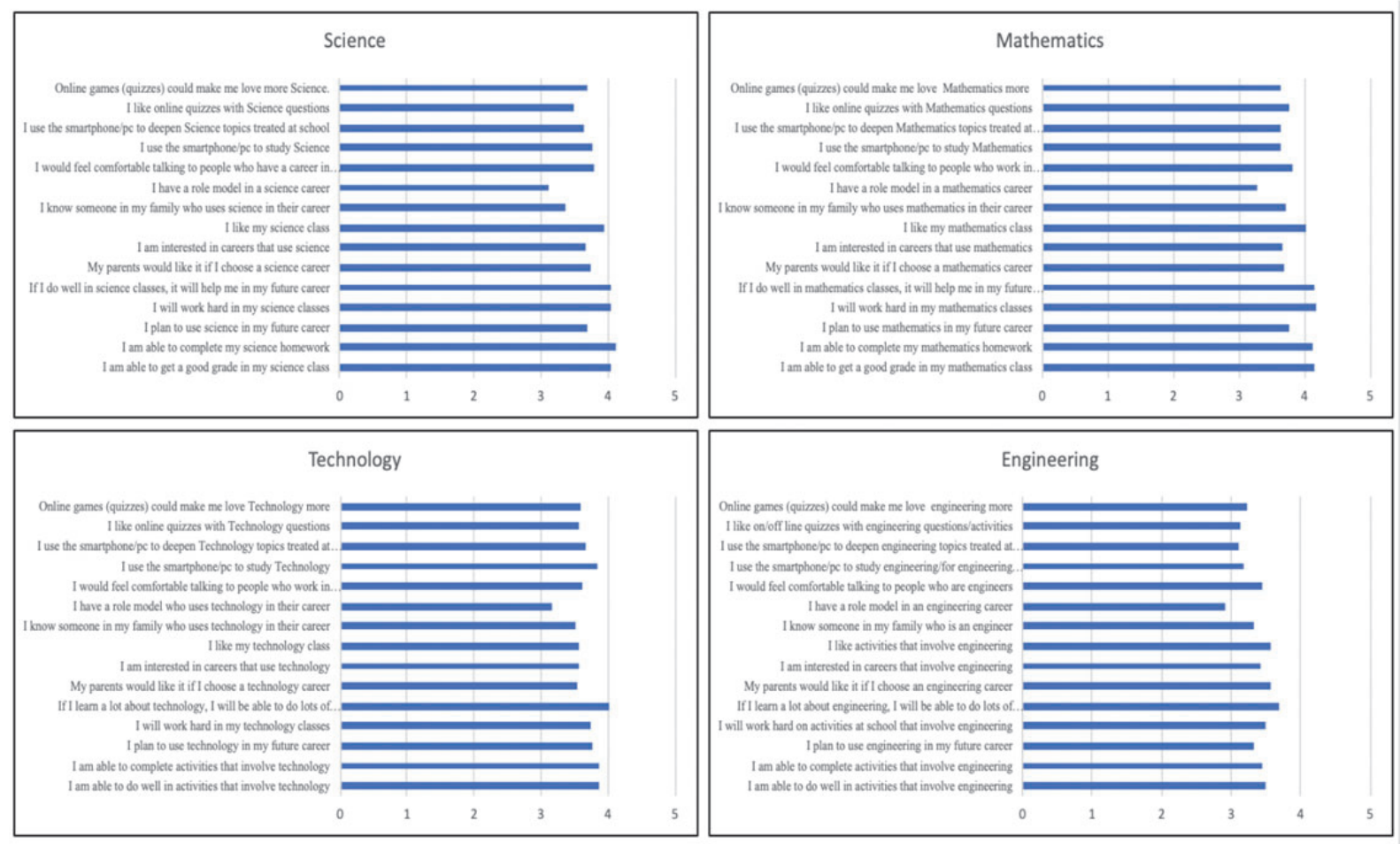

The results show a different scene in the different skills treated. The data underline a lower level of knowledge and interaction in Engineering and Technology compared to Mathematics and Science. Students' answer highlights also a lower level of score related to the question 'I have a role model in an engineering/technology/mathematics/science career' than the other questions proposed.

FIGURE 5

USE OF SMARTPHONE AND PC

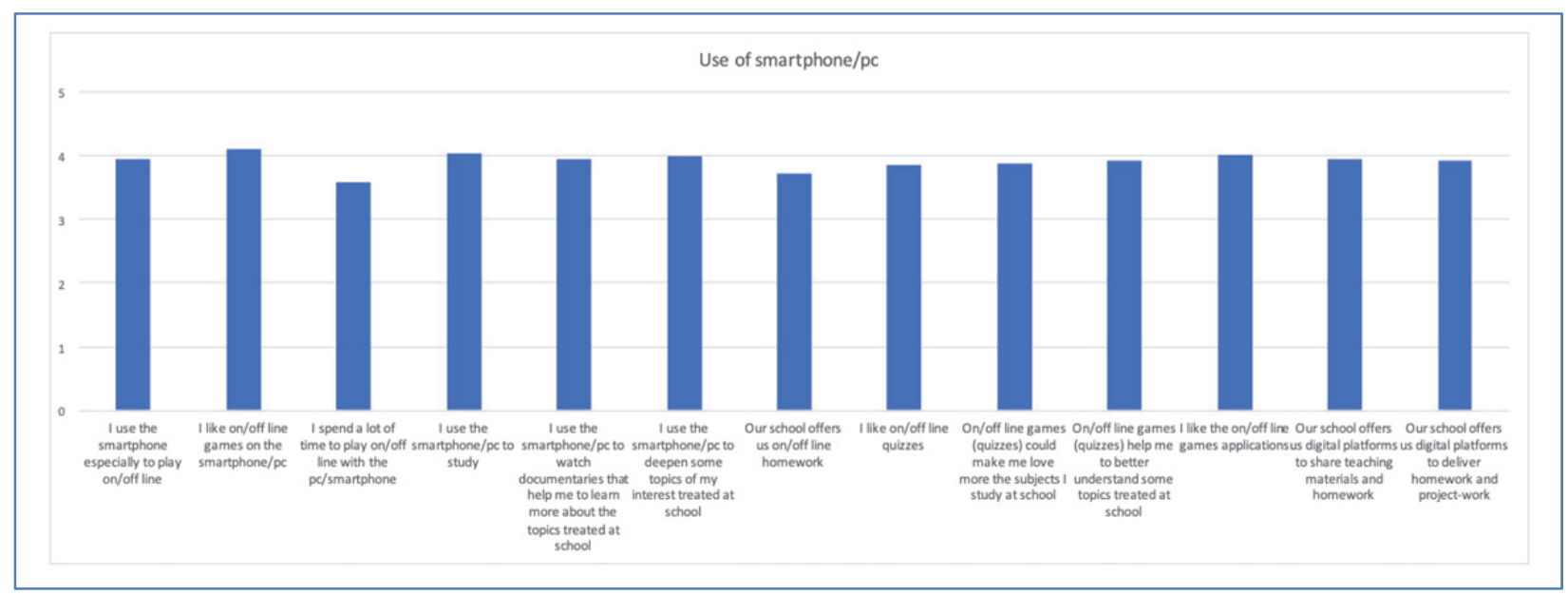

Another part of the questionnaire detects the students likely in using smart technologies to learn and to deepen school subjects (Figure 5). The results show a constant trend about a use of smartphone/pc. The 
two questions with a slightly lower score are 'I spend a lot of time to play on/off line with the pc/smartphone' and 'Our school offers us on/off line homework'.

FIGURE 6

KNOWLEDGE LEVEL

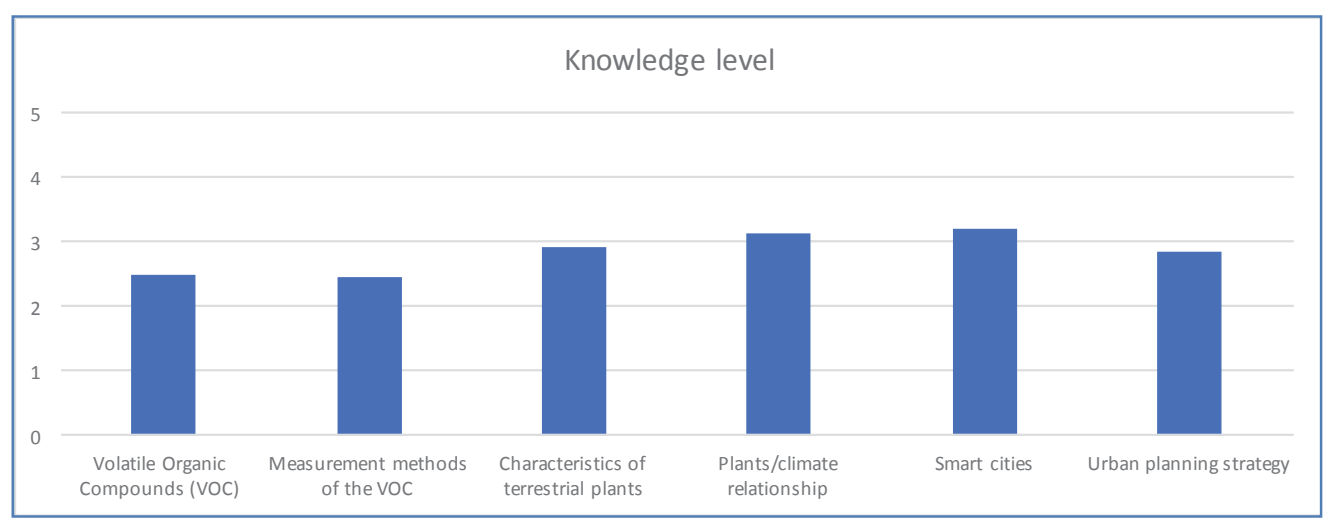

The last section of the questionnaire is designed to identify the knowledge level of STEM materials that will be treated in the framework of the DIGITgame project. The figure 6 shows a level of knowledge less than '4 - good' for all items. In particular, the figure highlights a lower score of knowledge level of 'VOC-Volatile Organic Compounds' and 'Measurement of the VOC'.

FIGURE 7

\section{SEARCHING INFORMATION BY SMARTPHONE/PC ABOUT STEM TOPICS}

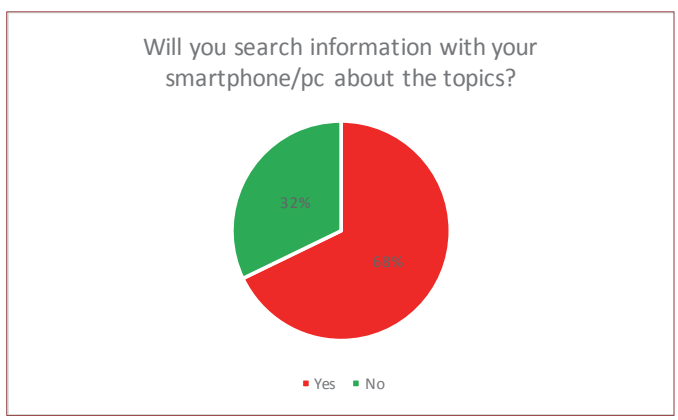

The section of the questionnaire that detected the level of knowledge of STEM underlines that more than $50 \%$ of students will search information about the topics that will be treated during DIGITgame project. The main topics that the students will be looking for are VOC and Smart Cities (See Figure 7).

This first survey, that will be continued during the DIGITgame project life, underlines that technology-supported didactic models as gaming, virtual laboratories, international collaborative projects, real-time formative assessment and skills-based assessment, have the potential to improve students' learning outcomes, including development of higher-order thinking skills, and to expand the range of learning opportunities made available to students (Prensky, 2010).

\section{CONCLUSION}

In the presented research, the respondents are students from 14 to 16 ages in Italy and Turkey. The schools are involved in the DIGITgame project in two different pilot activities: at the beginning of the project as developers of the videogame to increase their STEM knowledge and at the end of the 
development phase in order to support the other implementers schools involved to test and to improve the videogame.

The questionnaire was carried out before the beginning of the first pilot activity with the aim to collect information about the level of knowledge of STEM topics and the use of technology at school. The same questionnaire will be administrated at the same sample of students in order to understand and compare the level of knowledge at the end of the pilot activity (lessons and development of videogame).

The results of this first step of project show an interest and attitudes toward science and mathematics by the project students. While the results of the other skills analysed, engineering and technology, show a different framework. The score assigned to each question is lower than science and mathematics. In the Italian and Turkish schools the subject of engineering and technology are in the scholastic curriculum, but at the same time they are not specify as subject in the schools' hours. Otherwise mathematics and science are subjects itemise in the schools' hours. The lower score assigned to engineering and mathematics could be related to this aspects and students do not perceive they as specific subjects, despite being treated during school lessons.

The results are interesting and can be used in future research with integrated data collected also from other stakeholders, such as teachers, in order to compare some topics and understand the opinion of teachers in relation to students' skills.

DIGITgame project can represent a model to reinforce the learning of STEM concepts by technology applications for different target students. The objective of interest increasing is to promote student attitudes toward careers in science, technology, engineering, and mathematics.

Moreover, the work methodology taken by the Project team show the possibility of collaboration and support in an international framework, for promoting educational innovation.

\section{ACKNOWLEDGEMENT}

DIGITgame project is carrying out with the project partners in the framework of Erasmus + Programme 2017-1-IT02-KA201-026822. 


\section{REFERENCES}

Bennett, S., Maton, K., \& Kervin, L. (2008). The 'digital natives' debate: A critical review of the evidence. British journal of educational technology, 39(5), 775-786.

Kärkkäinen, K., \& Vincent-Lancrin, S. (2013). Sparking Innovation in STEM Education with Technology and Collaboration.

Kier, M. W., Blanchard, M. R., Osborne, J. W., \& Albert, J. L. (2014). The development of the STEM career interest survey (STEM-CIS). Research in Science Education, 44(3), 461-481.

Kilimci, S. (2009). Teacher training in some EU countries and Turkey: How similar are they? ProcediaSocial and Behavioral Sciences, 1(1), 1975-1980.

Klette, K. (2007). Trends in research on teaching and learning in schools: Didactics meets classroom studies. European Educational Research Journal, 6(2), 147-160.

Likert, R. (1932). A technique for the measurement of attitudes. Archives of psychology.

Prensky, M. R. (2010). Teaching digital natives: Partnering for real learning. Corwin Press.

Tapscott, D. (1998). Growing up digital (Vol. 302). San Francisco: McGraw-Hill Companies.

Wang, S. K., Hsu, H. Y., Campbell, T., Coster, D. C., \& Longhurst, M. (2014). An investigation of middle school science teachers and students use of technology inside and outside of classrooms: considering whether digital natives are more technology savvy than their teachers. Educational Technology Research and Development, 62(6), 637-662. 